\title{
Idiopathic peripheral neuropathy increases fall risk in a population-based cohort study of older adults
}

Jody L Riskowski ${ }^{1,2^{*}}$, Lien Quach¹, Brad Manor ${ }^{2,3}$, Hylton B Menz ${ }^{1,2,4}$, Lewis A Lipsitz ${ }^{1,2,3}$, Marian T Hannan ${ }^{1,2}$

From 3rd Congress of the International Foot and Ankle Biomechanics Community

Sydney, Australia. 11-13 April 2012

\section{Background}

Peripheral neuropathy (PN) is often associated with specific diseases; however, research suggests that idiopathic $\mathrm{PN}$ is prevalent in older adult populations [1]. Foot ulceration is the traditional medical concern with PN, but people with PN may also have disproportionately more falls [2]. Therefore, our objective was to evaluate associations between $\mathrm{PN}$ and prospectively-ascertained falls in older adults from the population-based MOBILIZE Boston Study.

\section{Materials and methods}

Participants included 760 MOBILIZE Boston Study members. PN was assessed using Semmes-Weinstein

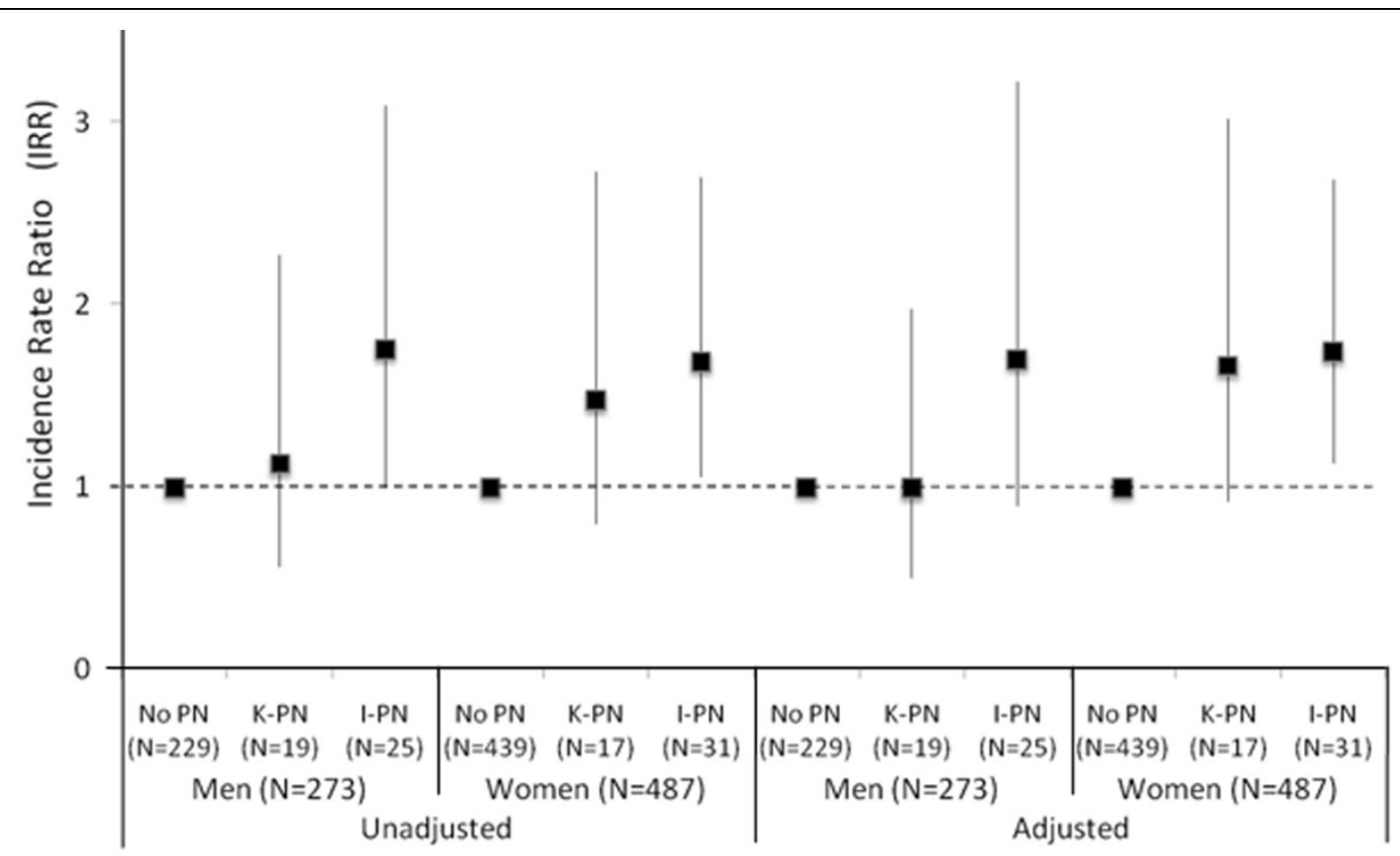

Figure 1 IRR between PN and falls

\footnotetext{
* Correspondence: JodyRiskowski@hsl.harvard.edu

1 Institute for Aging Research, Hebrew SeniorLife, Boston, Massachusetts

02131-1011, USA

Full list of author information is available at the end of the article
} 
monofilament testing [3], applying the modified Health ABC Study method $[4,5]$. Three PN status groups were defined: (i) no PN (referent), (ii) PN and known disease associated with PN (e.g., diabetes, autoimmune disease) (K-PN), and (iii) idiopathic PN (I-PN). Falls were tracked through monthly fall calendars over a mean 2.8 (range 1.4-4.3) year follow-up period. Unadjusted and adjusted (age, body mass index, physical activity, prior year fall, visual acuity, depression and number of medications) gender-specific negative binomial regression models determined associations between PN and falls.

\section{Results}

I-PN was associated with a higher fall incidence in men (incidence rate ratio [IRR] 1.76 [95\% confidence interval 1.00-3.09]; Figure 1) and women (1.69 [1.06-2.70]). These higher IRRs with I-PN persisted even after covariate adjustment in women (1.68 [1.09-2.60]) and men (1.70 [0.90-3.22]), with men's confidence interval widening. K-PN was not associated with an increased incidence of falling in men and had weak, non-significant effect in women.

\section{Conclusions}

Idiopathic $\mathrm{PN}$ is an independent fall risk factor for women and men, suggesting that PN assessments should be included in fall risk evaluations. Future work to investigate mechanisms through which $\mathrm{PN}$ increases fall risk and to evaluate interventions that target fall risk in individuals with $\mathrm{PN}$, such as insoles with low-grade vibrations [6], is needed.

\footnotetext{
Author details

${ }^{1}$ Institute for Aging Research, Hebrew SeniorLife, Boston, Massachusetts 02131-1011, USA. ${ }^{2}$ Harvard Medical School, Boston, Massachusetts 021156092, USA. ${ }^{3}$ Gerontology and Interdisciplinary Medicine and Biotechnology, Beth Israel Deaconess Medical Center, Boston, Massachusetts 02115-6092, USA. ${ }^{4}$ Musculoskeletal Research Centre, La Trobe University, Bundoora, Victoria 3086, Australia.
}

Published: 10 April 2012

\section{References}

1. Mold JW, Vesely SK, Keyl BA, Schenk JB, Roberts M: The prevalence, predictors, and consequences of peripheral sensory neuropathy in older patients. J Am Board Fam Pract 2004, 17:309-318.

2. Richardson JK, Hurvitz EA: Peripheral neuropathy: a true risk factor for falls. J Gerontol A Biol Sci Med Sci 1995, 50:M211-215.

3. Perkins BA, Olaleye D, Zinman B, Bril V: Simple screening tests for peripheral neuropathy in the diabetes clinic. Diabetes Care 2001, 24:250-256.

4. Strotmeyer ES, Cauley JA, Schwartz AV, de Rekeneire N, Resnick HE, Zmuda JM, Shorr Rl, Tylavsky FA, Vinik Al, Harris TB, et al: Reduced peripheral nerve function is related to lower hip BMD and calcaneal QUS in older white and black adults: the Health, Aging, and Body Composition Study. J Bone Miner Res 2006, 21:1803-1810.

5. Leveille SG, Jones RN, Kiely DK, Hausdorff JM, Shmerling RH, Guralnik JM, Kiel DP, Lipsitz LA, Bean JF: Chronic musculoskeletal pain and the occurrence of falls in an older population. JAMA 2009, 302:2214-2221.
6. Liu W, Lipsitz LA, Montero-Odasso M, Bean J, Kerrigan DC, Collins JJ: Noiseenhanced vibrotactile sensitivity in older adults, patients with stroke, and patients with diabetic neuropathy. Arch Phys Med Rehabil 2002, 83:171-176.

doi:10.1186/1757-1146-5-S1-P19

Cite this article as: Riskowski et al:: Idiopathic peripheral neuropathy increases fall risk in a population-based cohort study of older adults. Journal of Foot and Ankle Research 2012 5(Suppl 1):P19.

\section{Submit your next manuscript to BioMed Central and take full advantage of:}

- Convenient online submission

- Thorough peer review

- No space constraints or color figure charges

- Immediate publication on acceptance

- Inclusion in PubMed, CAS, Scopus and Google Scholar

- Research which is freely available for redistribution
C Biomed Central 